\title{
The Crucial Role of Nurses and Social Workers in Initiating End-of-Life Communication to Reduce Overtreatment in the Midst of the COVID-19 Pandemic
}

\author{
Chris Raftery ${ }^{a}$ Ebony Lewis ${ }^{b, c}$ Magnolia Cardonaa, \\ ${ }^{a}$ Gold Coast Hospital and Health Service, Southport, QLD, Australia; ${ }^{b}$ School of Public Health and Community \\ Medicine, The University of New South Wales, Randwick, NSW, Australia; 'School of Psychology, The University of \\ New South Wales, Randwick, NSW, Australia; ${ }^{d}$ Institute for Evidence-Based Healthcare, Bond University, \\ Robina, QLD, Australia
}

\section{Keywords}

End of life · Advanced care planning · Nurses $\cdot$ Allied health . COVID-19

\begin{abstract}
The large scale and rapid spread of the current COVID-19 pandemic has changed the way hospitals and other health services operate. Opportunities for patient-centered decision-making at the end of life are being jeopardized by a scarcity of health system resources. In response, the traditional doctor-initiated advanced care planning (ACP) for critical illness may also need to be readjusted. We propose nurse-led and allied health-led ACP discussions to ensure patient and family inclusion and understanding of the disease prognosis, prevention of overtreatment, and potential outcomes in crisis times. We highlight known barriers and list enablers, long-term and short-term opportunities to assist in the culture change.

๑) 2020 S. Karger AG, Basel
\end{abstract}

Medical technology and therapeutic advances have enabled us to have a prolonged life expectancy. However, in older people with advanced irreversible multimorbidity, prolonging suffering and impairing the quality of the remaining life may be questionable. The benefits of advanced care planning (ACP) in preventing unnecessarily aggressive and futile treatments near the end of life are well known among clinicians. Paradoxically, the uptake of ACP remains low and is a challenge globally.

Awareness of the need for documentation of patients' wishes has been heightened recently due to the coronavirus 2019 (COVID-19) pandemic, with high infection rates and mortality risk among older people and/or those with underlying chronic conditions. This rapid spread of COVID-19 is placing a strain on existing resources and limiting universal healthcare access in some health systems. Can the urgent end of life decision-making requirements during public emergencies such as restricted access to intensive care [1] or the appropriateness of resuscitation brought on by this pandemic [2] be met? We propose that this time of global crisis is an opportunity to rethink and reform the models of end-of-life care planning.

End-of-life discussions are usually doctor led [3] as their role in determining illness severity and leading decision-making is generally accepted [4]. More often than not, these discussions occur during an acute health crisis after a terminal diagnosis is clear, when patients and fam-

$\begin{aligned} & \text { karger@karger.com } \\ & \text { www.karger.com/ger }\end{aligned}$
Karger ${ }^{\prime /}$

Magnolia Cardona

Institute for Evidence-Based Healthcare, Faculty of Health Sciences and Medicine Bond University, 14 University Dr., Level 4, Building E

Robina, QLD 4226 (Australia)

mcardona@bond.edu.au 
Table 1. Current barriers and opportunities for non-medical staff engagement in initiating end-of-life discussions

\begin{tabular}{|c|c|}
\hline Barriers & Short-term opportunities \\
\hline $\begin{array}{l}\text { - A culture in healthcare that works against any change in } \\
\text { role delineation fueled by the social expectation that } \\
\text { doctors take the lead on prognostic disclosure and offers of } \\
\text { treatment options } \\
\text { - } \quad \text { Inadequate training and skills for initiation of such complex } \\
\text { end-of-life discussions } \\
\text { - Feeling that their input to the conversation is vague and } \\
\text { somewhat meaningless } \\
\text { - Lack of support, as a profession, in having end-of-life } \\
\text { responsibility within their scope of practice } \\
\text { Discomfort about implications due to the uncertainty of } \\
\text { whether taking a leading role in end-of-life discussions } \\
\text { could result in potential legal action by patients' families }\end{array}$ & 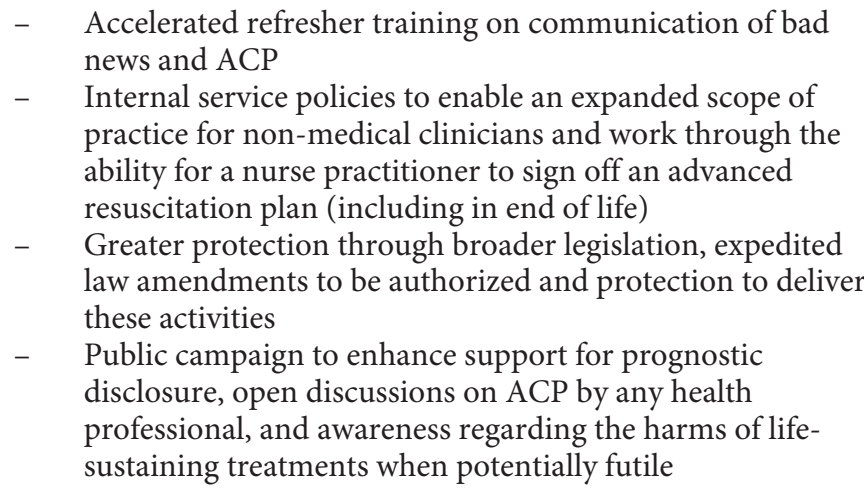 \\
\hline
\end{tabular}

ilies are at their most vulnerable, aiding the decision of whether to initiate or discontinue treatment [5]. Prognostic uncertainty, time constraints of busy routine practices, and limited access to psychosocial support following the breaking of bad news explain the lack of proactive ACP among doctors. However, public readiness is changing as recent research suggests that older members of the public more openly recognize the benefits of comfort care and are more willing to become involved in shared decisionmaking [6].

The unprecedented transmissibility of COVID-19 and the potential for high mortality are removing opportunities for patients and families to plan for future health care. The pandemic presents a challenge of time pressure for decisions because of the sudden increase in patient workload, resulting in the need to ration stretched resources to people with the best chances of survival [2]. While the concurrent economic crisis unfolds, the ethical dilemma of health care allocation requires urgent attention so that end-of-life care discussions including withholding lifesustaining treatments are not delayed. With the extensive media coverage and online information on the diagnosis, prognosis, and global spread of COVID-19 and the shortage of health personnel and medical supplies, the public is becoming aware of the possibility that restricted access to ideal healthcare is imminent. As in other pandemics, strict triage protocols for allocating health resources [7] may need to be introduced. Hence, management aligned with the patient's goals, wishes and preferences may no longer be feasible as equity of access to life-saving technology diminishes if the health system is overwhelmed [8]. However, there is still a chance to preserve dignity and provide supportive palliative care if end-of-life discussions are streamlined and widespread.

A strategy for the health system to adequately respond to the challenge of urgent decision-making is warranted. Delegating some of the responsibility of end-of-life discussions in the transition from curative to palliative care to other health professionals who feel ready and competent [9] may be one option. Non-medical clinicians, specifically nurses, can play a supportive role for patients and their families due to their ongoing interactions with patients and families at the bedside, which builds trust and rapport [5]. Nurses have a unique perspective of the personalized disease trajectory [10], which strengthens their credibility in end-of-life conversations. Nurses currently play three roles in end-of-life communication, including information broker, supporter, and advocate. Their role spans from translating technical medical details to advocating the patient and family's goals with the wider healthcare team $[4,5]$, through to navigating across the intricacies of the health system, and clarifying the need to prevent futile overtreatment. Many perceived and actual system and professional barriers have been identified internationally which prevent non-medical staff from becoming mainstream ACP champions (Table 1). Barriers, from level of competency through to system level legislation, can have an impact on effective engagement of endof-life discussions and in turn they can negatively affect the quality of end-of-life care for patients. However, potential solutions are also suggested both at the internal service level (palliative care and communication training [11] and mentoring) and at system-wide level (regulation, legislation, and accreditation) [4]. 
ACP nurse-led models of care implemented and evaluated in urban and rural settings [12] have shown promising results with good patient acceptability and understanding of its benefits [13]. An expanded role for allied health professionals including social workers in ACP has also been proposed due to their involvement in conflict resolution and advocacy duties [14] and advanced communication skills necessary to support this process. Given the chaotic hospital atmosphere, resource scarcity and doctor fatigue, and the burnout and isolation of those infected during the COVID-19 pandemic, the opportunity exists globally for end-of-life communication to be initiated by nurses and social workers if they are coupled with rapid educational updates and legal safeguards in routine practice (Table 1).

As an alternative to current models for the health system, a larger proportion of health professionals capable of initiating the conversation on values, preferences and prognostic disclosure of the inevitable deterioration could contribute to a reduction of overtreatment and deliver better quality of end-of-life care. The transition might start with expedited legislative amendment for the competency-ready nurse practitioners and clinical nurse consultants, especially those already working in palliative care, who are somewhat less impacted by day-to-day volume issues of COVID-19 and have more control over their workload.

With additional training, expanding the role of dedicated social workers into becoming effective initiators of endof-life discussions during this pandemic may also alleviate the pressure of doctors when facing the need to limit ICU admission or resuscitation. Importantly, consideration must be taken to not overburden specific individuals within occupational groups such as frontline workers who may be overwhelmed with direct clinical management. These subgroups should not be charged with the new role or responsibility in the current environment as their levels of distress, anxiety, depression, and insomnia are already known to be high during the pandemic $[15,16]$.

Among longer-term opportunities it may be worth considering the incorporation of palliative care as part of the annual licensure; recognizing specialized nurses [17]; placing a greater focus on palliative care in the standard undergraduate curriculum for social workers and nursing to increase confidence and competence in managing these difficult but necessary conversations; and endorsement associated with nursing registration each year in countries where relevant.

The final action moving forward is to have patients and families, hospital executives, and residential aged care be supportive of this cultural shift, recognizing the value of nurses and allied health workers' potential role in timely engagement of end-of-life discussions. The benefit of embracing this new model of care will be reduced non-beneficial overtreatment. With the literature suggesting that patients and families are more satisfied and in acceptance of decisions when a nurse is involved [5], we have likely already commenced this positive shift on this journey but this is the time to enact it more widely and normalize it.

\section{Conclusion}

The COVID-19 epidemic has placed an extraordinary burden on health systems worldwide and it has reignited awareness of the need for hastier decision-making near the end of life. This unprecedented high level of demand for intensive care services which cannot be satisfied may be an opportunity to explore an expanded scope of practice for key members of the broader healthcare team, i.e., nurses and social workers. They are suitably qualified and intimately familiar with the social and clinical circumstances to take on the role of initiators and facilitators of end-of-life discussions with patients and families. With targeted education, legislative support, and public awareness, it may be possible to implement a change to the healthcare culture that improves the understanding of quality end of life care, accepts the role of palliative care, and normalizes the need to withhold or withdraw futile life-sustaining therapies for people who do not have prospects of survival. Concurrent evaluation of the effectiveness of this model of care is encouraged.

\section{Conflict of Interest Statement}

The authors have no conflict of interests to declare.

\section{Funding Sources}

None.

\section{Author Contributions}

M.C. conceived the idea, structured the first draft, supervised the process, and refined the last version of this paper. C.R. contributed substantially to the first draft. C.R. and E.L. contributed comments and paragraphs for subsequent versions with clinical and intellectual input. All of the authors approved the final version of this work. 


\section{References}

1 Fink SL. Worst case: rethinking tertiary triage protocols in pandemics and other health emergencies. Crit Care. 2010;14(1):103.

2 Curtis JR, Kross EK, Stapleton RD. The Importance of Addressing Advance Care Planning and Decisions About Do-Not-Resuscitate Orders During Novel Coronavirus 2019 (COVID-19). JAMA. 2020 Mar. https://doi. org/10.1001/jama.2020.4894.

3 Bally KW, Krones T, Jox RJ. Advance Care Planning for People with Dementia: The Role of General Practitioners. Gerontology. 2020; 66(1):40-6.

4 Hebert K, Moore H, Rooney J. The Nurse Advocate in End-of-Life Care. Ochsner J. 2011; 11(4):325-9.

5 Adams JA, Bailey DE Jr, Anderson RA, Docherty SL. Nursing roles and strategies in endof-life decision making in acute care: a systematic review of the literature. Nurs Res Pract. 2011;2011:527834.

6 Cardona M, Lewis E, Shanmugam S, Nicholson M, Williamson M, Hanly L, et al. Dissonance on perceptions of end-of-life needs between health-care providers and members of the public: quantitative cross-sectional surveys. Australas J Ageing. 2019 Sep;38(3):e7584.
7 Christian MD, Hawryluck L, Wax RS, Cook T, Lazar NM, Herridge MS, et al. Development of a triage protocol for critical care during an influenza pandemic. CMAJ. 2006 Nov; 175(11):1377-81.

8 Biddison LD, Berkowitz KA, Courtney B, De Jong CM, Devereaux AV, Kissoon N, et al.; Task Force for Mass Critical Care; Task Force for Mass Critical Care. Ethical considerations: care of the critically ill and injured during pandemics and disasters: CHEST consensus statement. Chest. 2014 Oct;146(4 Suppl): e145S-55S.

9 Ke LS, Huang X, O'Connor M, Lee S. Nurses' views regarding implementing advance care planning for older people: a systematic review and synthesis of qualitative studies. J Clin Nurs. 2015 Aug;24(15-16):2057-73.

10 Martin B, Koesel N. Nurses' role in clarifying goals in the intensive care unit. Crit Care Nurse. 2010 Jun;30(3):64-73.

11 Evenblij K, Ten Koppel M, Smets T, Widdershoven GA, Onwuteaka-Philipsen BD, Pasman HR. Are care staff equipped for end-oflife communication? A cross-sectional study in long-term care facilities to identify determinants of self-efficacy. BMC Palliat Care. 2019 Jan; 18(1):1-1.

12 Sinclair C, Auret KA, Evans SF, Williamson F, Dormer S, Wilkinson A, et al. Advance care planning uptake among patients with severe lung disease: a randomised patient preference trial of a nurse-led, facilitated advance care planning intervention. BMJ Open. 2017 Feb; 7(2):e013415.
13 Miller H, Tan J, Clayton JM, Meller A, Hermiz $\mathrm{O}, \mathrm{Zwar} \mathrm{N}$, et al. Patient experiences of nursefacilitated advance care planning in a general practice setting: a qualitative study. $\mathrm{BMC} \mathrm{Pal}-$ liat Care. 2019 Mar; 18(1):25.

14 Wang CW, Chan CL, Chow AY. Social workers' involvement in advance care planning: a systematic narrative review. BMC Palliat Care. 2017 Jul; 17(1):5.

15 Lai J, Ma S, Wang Y, Cai Z, Hu J, Wei N, et al. Factors Associated With Mental Health Outcomes Among Health Care Workers Exposed to Coronavirus Disease 2019. JAMA Netw Open. 2020 Mar;3(3):e203976-76.

16 Pappa S, Ntella V, Giannakas T, Giannakoulis VG, Papoutsi E, Katsaounou P. Prevalence of depression, anxiety, and insomnia among healthcare workers during the COVID-19 pandemic: a systematic review and metaanalysis. Brain Behav Immun. 2020. doi: 10.1016/j.bbi.2020.05.026.

17 American Nurses Association. Call for action: nurses lead and transform palliative care. 2017. Available from: https://www.nursingworld.org/ 497158/globalassets/practiceandpolicy/health-policy/palliativecareprofessionalissuespanelcallforaction.pdf. 